CLEO-Europe/IQEC 2007, Munich $17-22$ June (accepted)

\title{
Femtosecond Laser-Induced Forward Transfer (LIFT): A Technique for Versatile Micro-Printing Applications.
}

\author{
D. P. Banks, C. Grivas, R. W. Eason \\ Optoelectronics Research Centre, University of Southampton, Southampton SO17 IBJ, United Kingdom \\ I. Zergioti \\ Physics Department, National Technical University of Athens, 15780 Zografou, Greece
}

The Laser-Induced Forward Transfer (LIFT) method ${ }^{1}$ exists as a relatively simple and versatile additive surface micropatterning technology. Material is transferred from a supported thin film to a receiver substrate by irradiating the rear side of the film with a single laser pulse. Typically transfer is effected either through melting through of the source film or by ablation of the film at a constrained interface with a resultant pressure build-up propelling a piece of the film to the receiver. Both of these processes have inherent advantages and disadvantages; by melting the source film during transfer, sub-laser spot size features can be produced, but the choice of available materials is reduced and control of deposit morphology is limited. Ablation-driven transfer is less material selective but resultant deposits are typically broken during transfer and scattered over relatively large areas.
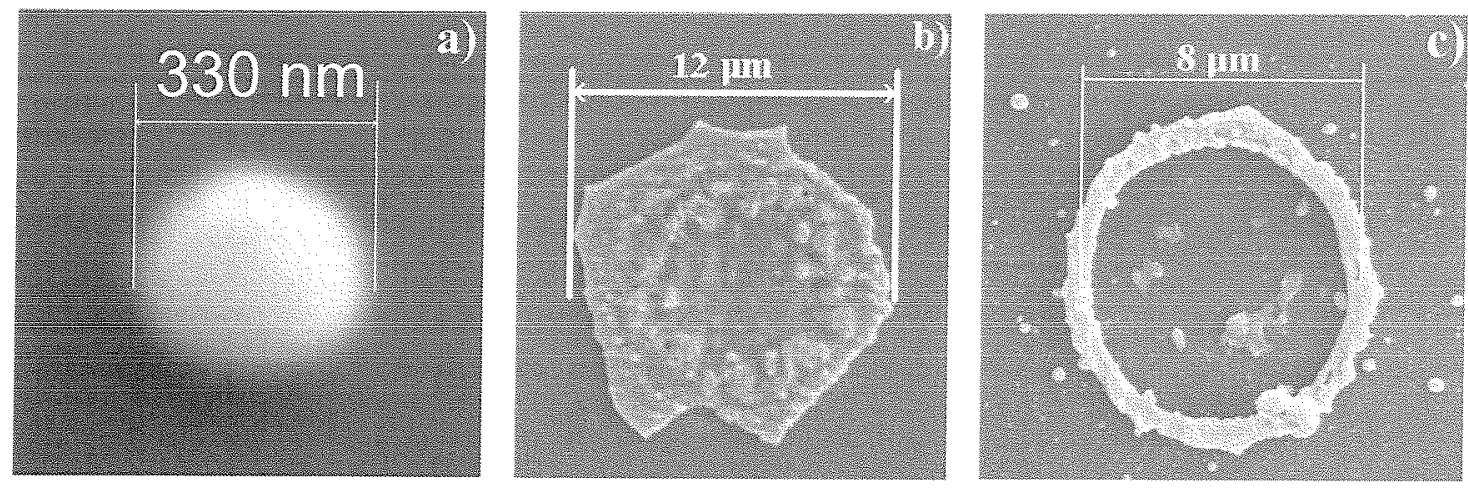

Fig. 1 SEM micrographs of $\mathrm{Cr}$ deposited by femtosecond LIFT: (a) a sub-wavelength nanodroplet, (b) a pellet of intact thin film material, and (c) a ring-shaped deposit.

As LIFT is essentially a thermal process, it is possible to exploit the reduction in heat diffusion inherent with femtosecond light-matter interactions to extend the capabilities of the technique to overcome some of the limitations of nanosecond LIFT. We will present the latest results from our ongoing study of femtosecond LIFT. These results will include:

- the smallest features so far obtained using LIFT (Fig. 1(a)) ${ }^{2}$. By just melting through a very thin film, controlling the laser fluence such that minimal ablation occurs at the constrained interface, sub-spot size and sub-wavelength $(\lambda=800 \mathrm{~nm})$ nanodroplets of $\mathrm{Cr}$ have been produced with reproducible diameters of below $350 \mathrm{~nm}$.

- the first demonstration of forward transfer of segments of intact source film material that have not melted or shattered during transfer (Fig. 1(b)). Using multiple sub-threshold pulses to progressively weaken a section of film before transfer, it is possible to print pellets of film relatively intact. Such a capability represents an exciting extension of LIFT to print materials that have not previously been available.

- defining the shape of deposits using the transverse laser spatial profile. Combining the reduced thermal diffusion available with femtosecond LIFT with spatial shaping of the laser pulse, high resolution patterns can be deposited in a single transfer event (Fig. 1(c)).

\section{References}

1. J. Bohandy, B. F. Kim, and F. J. Adrian, "Metal deposition from a supported metal film using an excimer laser", J. Appl. Phys. 60(4), 1538-1539 (1986).

2. D. P. Banks, C. Grivas, J. D. Mills, I. Zergioti, and R. W. Eason, "Nanodroplets deposited in microarrays by femtosecond Ti:sapphire laser-induced forward transfer", Appl. Phys. Lett. 89, 193107 (2006). 